\title{
High-Precision Measurement of the Hypertriton Mass
}

\author{
Patrick Achenbach*, Sebastian Bleser, Josef Pochodzalla*, Marcell Steinen \\ Institut für Kernphysik, Johannes Gutenberg-Universität Mainz, Germany \\ and Helmholtz Institute Mainz, Germany \\ E-mail: achenbach@uni-mainz.de, pochodza@uni-mainz.de
}

In recent years, the high-precision technique of decay-pion spectroscopy at the Mainz Microtron (MAMI), using high-resolution magnetic spectrometers, contributed to the accurate determination of the $\Lambda$ binding energy of ${ }_{\Lambda}^{4} \mathrm{H}$. For the lightest hypernucleus, the hypertriton, the binding energy is known only from measurements carried out with nuclear emulsions. An account is given on the potential of observing the hypertriton with decay-pion spectroscopy from fragmentation of light target nuclei. Statistical decay calculations suggest that lithium is the optimal target material to observe hypertriton decays under relative clean conditions with only few other light hyperfragments being produced. Finally, a high-precision measurement of the hypertriton mass at MAMI is proposed.

XVII International Conference on Hadron Spectroscopy and Structure - Hadron2017

25-29 September, 2017

University of Salamanca, Salamanca, Spain

${ }^{*}$ Speakers. 


\section{Binding Energy of Hypertriton}

Our knowledge of the $\Lambda N$ interaction is based on measurements of observables such as $\Lambda$ binding energies $B_{\Lambda}$, excitation energies for particle-bound states, spins, lifetimes, and decay branching ratios. Most binding energies of light hypernuclei $(A<14)$ are known from nuclear emulsion experiments [1,2], in which they were determined by analyzing the kinetic energies of products from weak pionic decays. Only recently, $B_{\Lambda}$ values of light hypernuclei could have been determined by electron scattering experiments using high-resolution magnetic spectrometers, e.g. with the method of decay-pion spectroscopy at the Mainz Microtron (MAMI) for the binding energy of ${ }_{\Lambda}^{4} \mathrm{H}$ [3].
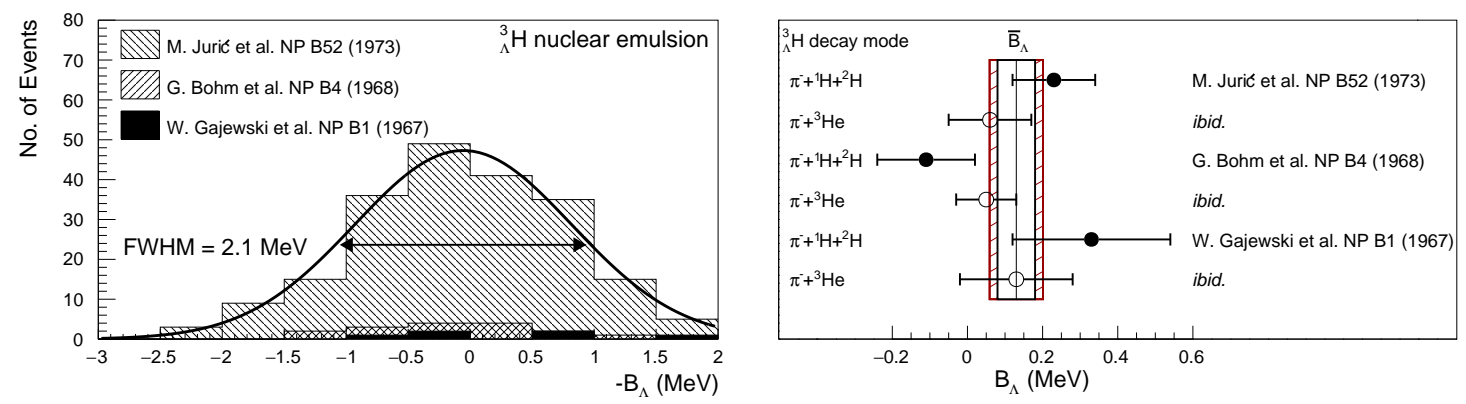

Figure 1: Left: Distribution of binding energies of hypertriton determined from pionic decays observed in emulsion experiments $[4,5,6]$. A Gaussian function was fitted to the distribution for illustration of the spread of the values, resulting in a FWHM of $2.1 \mathrm{MeV}$. Right: Binding energy data, separated into two-body and three-body pionic decays $[4,5,6]$ (with statistical errors only). The total number of events was $N=204$ and the mean value was evaluated in ref. [4] to be $\bar{B}_{\Lambda}=0.13 \pm 0.05 \mathrm{MeV}$ (statistical and total uncertainties including an estimate of the systematic error [1]).

Despite the small systematic error assigned to the emulsion measurements [1], it was shown recently [7] that for $p$-shell hypernuclei a discrepancy in the range of 400 to $800 \mathrm{keV}$ between the emulsion data [2] and those obtained with the $\left(\pi^{+}, K^{+}\right)$reaction [8] exists. This discrepancy could be partially resolved by a correction of the emulsion data for ${ }_{\Lambda}^{12} \mathrm{C}$ which served as a calibration point for $\left(\pi^{+}, K^{+}\right)$measurements. A similar observation was made in a survey of existing hypernuclei data taking also the FINUDA measurements into account [9]. Even after the re-calibration of the $\left(\pi^{+}, K^{+}\right)$spectrometer data with respect to the FINUDA data there remained substantial differences between emulsion studies and electronic experiments with a significant spread from $-700 \mathrm{keV}$ to $+200 \mathrm{keV}$ for individual hypernuclei. Both studies suggest that the emulsion data could have larger systematic uncertainties than published and that these differ dependent on the hypernucleus.

The first observation of hyperfragments by the technique of decay-pion spectroscopy with a high-resolution magnetic spectrometer was performed in 2012 at the Mainz Microtron MAMI [3]. The technique used by the A1 Collaboration was to measure the binding energy of light hypernuclei produced in a multi-step strangeness production, nuclear fragmentation and pionic weak decay reaction chain following the $\left(e, e^{\prime} K^{+}\right)$reaction on a ${ }^{9} \mathrm{Be}$ target. In 2014 , an extended measurement campaign was performed with improved control over systematic effects and confirming the measurement with two spectrometers at the same time [10]. The $\Lambda$ binding energy resolution was designed to be $\delta_{B} \leq 100 \mathrm{keV}$, the highest resolution in hypernuclear spectroscopy using magnetic spectrometers. 
Especially unfortunate is the fact, that for the simplest hypernucleus known, the hypertriton, so far no reliable magnetic spectrometer data for its binding energy is available, i.e. no crosscheck for the emulsion studies exists. The binding energy values from emulsion experiments were compiled by refs. $[4,5,6]$. The distribution of binding energies of hypertriton determined from pionic twobody and three-body decays is shown in Fig. 1 (left). The mean $B_{\Lambda}$ value of the 176 events of the two-body mode was $0.07 \mathrm{MeV}$ and of the 46 events of the three-body mode was $0.13 \mathrm{MeV}$. A mean value was evaluated using both decay modes to be $B_{\Lambda}=0.13 \pm 0.05 \mathrm{MeV}$ [4]. The $B_{\Lambda}$ value evaluated by ref. [5] differs from this by $\Delta B_{\Lambda}=0.14 \pm 0.11 \mathrm{MeV}$. The published $B_{\Lambda}$ values are shown in Fig. 1 (right).

Like in the case of deuterium for conventional baryon interactions, the hypertriton provides several important benchmarks for any strong interaction theory dealing with strange baryons. As the hypertriton is so little bound, its lifetime and its $\Lambda$ binding energy are expected to be intimately related $[11,12]$. Its very small binding energy as observed by the emulsion technique implies that the bound $\Lambda$ has an extended wave function and should have properties similar to the free $\Lambda$ [13]. In contrast, its lifetime observed in heavy-ion collisions is $30-40 \%$ shorter than the free $\Lambda$ lifetime $[14,15,16]$ leading to a strongly debated puzzle in hypernuclear physics, as demonstrated by the "Topical Session on the Lifetime Measurement of the Hypertriton" during the HYP2015 conference [17]. This situation calls for a precision measurement of the hypertriton binding energy.

Such a measurement gains further relevance by recent hypernuclear studies in ultra-relativistic heavy ion collisions. In such collisions the formation of light fragments from the hot participants zone can be described either by statistical or by coalescence processes. A statistical scenario assumes that in a high energy collision all hadrons follow equilibrium distributions at freeze-out. The production yield for a fragment is a function of the ratio between chemical freeze-out temperature and the mass of the fragment. It is insensitive to the internal structure of the nucleus. This is opposite to the case of a coalescence of baryons into nuclei, where the yield depends on the phase space density and on the individual coalescence radius, which reflects the internal wave functions of the composite fragment.

The large size of the hypertriton makes it a unique probe for the fragment formation process in ultra-relativistic heavy ion collisions. In ref. [18] the strangeness population factor was introduced in terms of the ratio of the production yields, $S_{3}=Y\left({ }_{\Lambda}^{3} \mathrm{H}\right) / Y\left({ }^{3} \mathrm{He}\right) \cdot Y(\mathrm{p}) / Y(\Lambda)$. The value of $S_{3}=0.36 \pm 0.26$ found by the E864 Collaboration indicated a penalty factor for attaching a hyperon to a cluster [18]. Based on a coalescence picture, it was suggested that the large spatial extent of hypertriton is a possible reason for this low value of $S_{3}$. Early data from the STAR Collaboration [14] and also later data from an energy scan [19] indicate a strong rise of $S_{3}$ with increasing $\sqrt{s_{N N}}$. On the other hand, thermal models predicted a rather weak energy dependence of $S_{3}$, while a coalescence picture predicted even a decrease of $S_{3}$ in the $\sqrt{s_{N N}}$ range between $3-20 \mathrm{GeV}$ [20]. However, at LHC energies the recent data from the ALICE Collaboration show again a decrease of $S_{3}$ [16]. It remains to be seen whether a consistent description of $S_{3}$ in the AGS and LHC energy range will be possible within a single picture or whether several effects of partonic or of hadronic nature - need to be considered. Consequently, even if the small binding energy of hypertriton will be confirmed by the new measurement proposed at MAMI, it is certainly desirable to improve its statistical and, in particular, its systematic uncertainty in order to guide precise structure calculations. 


\section{Hypertriton Formation, Stopping, and Decay}

Most striking of the hypernuclei experiments at MAMI in 2012 and 2014 was the fact that only one pion line was clearly observed. To understand this observation and to arrive at a prediction for hypertriton production in a decay-pion experiment at MAMI, a chain of models and Monte Carlo simulations had to be combined. The estimate includes (i) the elementary reaction kinematics and cross sections for the strangeness production process, (ii) a statistical decay model for the fragmentation mechanism, (iii) the stopping probabilities for the hyperfragments, (iv) the two-body decay branching ratios of the pionic decay, and finally the spectrometer acceptances, efficiencies, and survival fractions for the pion detection. This estimate was first outlined in ref. [21] with extensions and updates found in ref. [22].

\subsection{Compound Hypernucleus Formation}

The elementary $K \Lambda$ photoproduction of strangeness is well studied and differential cross sections of the order of $0.2 \mu \mathrm{b} / \mathrm{sr}<d \sigma / d \Omega<0.35 \mu \mathrm{b} / \mathrm{sr}$ at small kaon angles $\theta<30^{\circ}$ are known from experiments at Jefferson Lab [23]. Also, the electroproduction of quasi-free and bound states from light nuclear targets was studied comprehensively. Differential cross sections for electroproduction of $K^{+} \Lambda$ and $K^{+} \Sigma^{0}$ final states on $A=1-4,12$ targets are given in ref. [24]. Electroproduction processes involve the exchange of a virtual photon $\gamma^{*}$ between projectile and target. The cross section falls with the inverse of the invariant momentum transfer, $Q^{2}$, and a virtual photon flux factor $\Gamma$ can be defined for which $\Gamma \propto \alpha / Q^{2}$. With the scattered electron undetected the integral over $Q^{2}$ of the electroproduction cross section is dominated by reaction kinematics near the photon point at $Q^{2}=0$.

\begin{tabular}{lrlr}
\hline \hline${ }_{\Lambda}^{6} \mathrm{He}^{*}$ compound & $E_{\mathrm{thr}}$ & ${ }_{\Lambda}^{7} \mathrm{He}^{*}$ compound & $E_{\mathrm{thr}}$ \\
\hline${ }_{\Lambda}^{3} \mathrm{H}+\mathrm{t}$ & 20.75 & ${ }_{\Lambda}^{3} \mathrm{H}+\mathrm{n}+\mathrm{t}$ & 23.79 \\
${ }_{\Lambda}^{3} \mathrm{H}+\mathrm{n}+\mathrm{d}$ & 27.01 & ${ }_{\Lambda}^{3} \mathrm{H}+{ }^{4} \mathrm{H}$ & 26.69 \\
${ }_{\Lambda}^{3} \mathrm{H}+\mathrm{n}+\mathrm{n}+\mathrm{p}$ & 29.24 & ${ }_{\Lambda}^{3} \mathrm{H}+\mathrm{n}+\mathrm{n}+\mathrm{d}$ & 30.05 \\
& & ${ }_{\Lambda}^{3} \mathrm{H}+\mathrm{n}+\mathrm{n}+\mathrm{n}+\mathrm{p}$ & 32.27 \\
\hline${ }_{\Lambda}^{4} \mathrm{H}+\mathrm{d}$ & 18.84 & ${ }_{\Lambda}^{4} \mathrm{H}+\mathrm{t}$ & 15.63 \\
${ }_{\Lambda}^{4} \mathrm{H}+\mathrm{n}+\mathrm{p}$ & 21.07 & ${ }_{\Lambda}^{4} \mathrm{H}+\mathrm{n}+\mathrm{d}$ & 21.88 \\
& & ${ }_{\Lambda}^{4} \mathrm{H}+\mathrm{n}+\mathrm{n}+\mathrm{p}$ & 24.11 \\
\hline & & ${ }_{\Lambda}^{6} \mathrm{H}+\mathrm{p}$ & 23.95 \\
\hline & & ${ }_{\Lambda}^{6} \mathrm{He}+\mathrm{n}$ & 3.04 \\
\hline \hline
\end{tabular}

Table 1: Energy thresholds $E_{\mathrm{thr}}$ in $\mathrm{MeV}$ for the break-up of a compound hypernucleus from ${ }^{6,7} \operatorname{Li}\left(e, e^{\prime} K^{+}\right)$ into one lighter hyperfragment plus up to four nucleons or nuclear fragments. Thresholds were calculated for ${ }_{\Lambda}^{6} \mathrm{He}^{*}$ (left) and ${ }_{\Lambda}^{7} \mathrm{He}^{*}$ (right). For each hyperfragment the break-up channels are ordered by the energy threshold. ${ }_{\Lambda}^{4} \mathrm{He}$ and ${ }_{\Lambda}^{5} \mathrm{He}$ are not tabulated because no two-body $\pi^{-}$decay channels exist.

Experimental information on the observation of hyperfragments from different target nuclei or information on different hyperfragment yields from a given target can be used to test theoretical 
models of nuclear structure and to study the microscopic reaction process.

In the compound hypernucleus model energy and momentum is transfered to a quasi-free produced $\Lambda$ that scatters either on the residual nucleus in a virtual process or re-scatters off protons or neutrons in a real process from an independent target nucleus, and finally can lead to continuum excited states of ${ }_{\Lambda}^{9} \mathrm{Li}^{*},{ }_{\Lambda}^{8} \mathrm{Li}^{*}$, and ${ }_{\Lambda}^{8} \mathrm{He}^{*}$, etc. The formation of compound hypernuclei were used to explain the abundant production of ${ }_{\Lambda}^{4} \mathrm{H}$ observed in stopped- $\mathrm{K}^{-}$absorption on light nuclei [25].

One possibility to model the formation process is the introduction of a sticking probability as for example in [26], assuming harmonic oscillator wave functions for the bound hyperon. For the estimate of a minimum formation probability, the sticking probability was modeled with an exponentially falling dependence on the momentum difference between hyperon and nuclear core including the Fermi Gas distribution of the nuclei. In the quasi-free electroproduction process on light targets the excitation energies are in the range of $10-50 \mathrm{MeV}$ according to measured missing mass distributions at Jefferson Lab [24]. These distributions were folded with the sticking probability of the hyperon in order to determine the distribution of the initial excitation energy $f\left(E_{\text {exc }}\right)$. Guided by ref. [26] we adopted a sticking probability $\propto \exp \left(p_{\Lambda} / p_{0}\right)$ with $p_{0}=100 \mathrm{MeV} / c$. Within a simplified picture, the excitation energy of the equilibrated compound hypersystem can be estimated assuming a hole excitation by the proton converted into a $\Lambda$ and for the energy of the captured hyperon $E_{\mathrm{exc}} \approx \frac{1}{2}\left(V_{N}-B_{N}\right)+p_{F}^{2} / 2 M_{\Lambda} \sim(15+20) \mathrm{MeV}$. Indeed, $f\left(E_{\mathrm{exc}}\right)$ peaks around the value of $35 \mathrm{MeV}$. In case of an additional knockout of a nucleon prior to the $\Lambda$ capture, the additional hole excitation results in a $15 \mathrm{MeV}$ higher excitation energy.

Since the composition and the excitation energy of an initially produced compound hypernucleus from the target of mass number $A$ is not known, the calculations were performed as a function of the energy $E_{\text {exc }}=E_{\Lambda}+B_{\Lambda}$ with $B_{\Lambda}$ being the $\Lambda$ binding energy in the ground state of the compound hypernucleus. The excitation energy could be significantly reduced when the $\Lambda$ would scatter, e.g. an energetic nucleon $N^{\prime}$ could be knocked out of the target nucleus when the $\Lambda$ forms a compound hypernucleus with mass number $A-1$. Also an initial $\Sigma$ production followed by $\Sigma N \rightarrow \Lambda N^{\prime}$ conversion could contribute to the production of $A-1$ compound hypernuclei.

\subsection{Hyperfragment Formation and Stopping}

The statistical distribution of energy in the competition between decay channels can be used to predict the fragmentation yields for the different hyperfragments [27] following the Fermi break-up model [28]. In the case of conventional nuclear fragments, their ground states masses and particlestable excited states were taken into account. Also for hypernuclei the masses and known excited states were used. In the model all possible break-up channels are considered, which satisfy mass number, strangeness, charge, energy and momentum conservation.

For light nuclei with mass numbers $A \leq 13$, even a relatively small excitation energy could be comparable to the $\Lambda$ binding energy or be above nucleon or cluster emission thresholds. It is assumed that the principal mechanism of de-excitation of the compound hypernucleus is the simultaneous break-up into a lighter hypernucleus plus one or more nucleons or nuclear fragments such as $\mathrm{d}, \mathrm{t},{ }^{3} \mathrm{He}$, or $\alpha$, with all fragments being in ground or low excited states [29]. Table 1 lists the energy thresholds for possible fragmentation reactions in Li target nuclei.

The hyperfragments will loose kinetic energy in the target and dependent on their charge state, mass, and initial energy a certain fraction will be stopped inside the target before they decay. 


\subsection{Hyperfragment Decay}

The predictions for the hyperfragmentation yields from for ${ }^{6,7} \mathrm{Li}$ target nuclei with subsequent two-body pionic decay at rest are shown in Fig. 2. The predictions include the probabilities $D_{C}\left({ }_{\Lambda}^{A} Z, E_{\text {exc }}\right)$ of forming a hyperfragment ${ }_{\Lambda}^{A} Z$ from the compound hypernucleus $C$ with an excitation energy $E_{\text {exc }}$, the stopping probability $S$, and the two-body $\pi^{-}$decay branching ratio $\operatorname{Br}\left({ }_{\Lambda}^{A} Z\right)=\Gamma\left({ }^{A}(Z+1)+\pi^{-}\right) / \Gamma$ of the hyperfragment. Some of the branching ratios are not measured, so that estimates had to be used. Unfortunately, most of these branching ratios are not measured for nuclei with $A>4$. In cases where no experimental information is available, theoretical predictions or estimates based on the general trend were used. While this may introduce large uncertainties for heavy helium hypernuclei, the prediction for the relative yields of hypertriton and ${ }_{\Lambda}^{4} \mathrm{H}$ are not affected by such uncertainties. Table 2 lists the fragmentation yields for three different $E_{\text {exc }}$, namely below, close to, and above the maximum predicted yields.

$D_{C}$ and $P(x)$ in units of $10^{-4}, x$ in units of $\mathrm{mm}$

\begin{tabular}{|c|c|c|c|c|c|c|c|c|c|}
\hline \multicolumn{10}{|c|}{${ }_{\Lambda}^{6} \mathrm{He}^{*}$ compound from ${ }^{6} \mathrm{Li}$ target } \\
\hline & \multicolumn{3}{|c|}{$E_{\mathrm{exc}}=20 \mathrm{MeV}$} & \multicolumn{3}{|c|}{$E_{\mathrm{exc}}=40 \mathrm{MeV}$} & \multicolumn{3}{|c|}{$E_{\mathrm{exc}}=60 \mathrm{MeV}$} \\
\hline${ }_{\Lambda}^{A} Z$ & $D_{C}$ & $P(0.25)$ & $P(1.5)$ & $D_{C}$ & $P(0.25)$ & $P(1.5)$ & $D_{C}$ & $P(0.25)$ & $P(1.5)$ \\
\hline${ }_{\Lambda}^{3} \mathrm{H}$ & - & - & - & 27. & 1.4 & 3.7 & 17. & 1.0 & 2.1 \\
\hline${ }_{\Lambda}^{4} \mathrm{H}$ & 38. & 8.1 & 17. & 56. & 11. & 22. & 20. & 3.8 & 7.5 \\
\hline \multicolumn{10}{|c|}{${ }_{\Lambda}^{7} \mathrm{He}^{*}$ compound from ${ }^{7} \mathrm{Li}$ target } \\
\hline & \multicolumn{3}{|c|}{$E_{\mathrm{exc}}=20 \mathrm{MeV}$} & \multicolumn{3}{|c|}{$E_{\text {exc }}=40 \mathrm{MeV}$} & \multicolumn{3}{|c|}{$E_{\mathrm{exc}}=60 \mathrm{MeV}$} \\
\hline${ }_{\Lambda}^{A} Z$ & $D_{C}$ & $P(0.25)$ & $P(1.5)$ & $D_{C}$ & $P(0.25)$ & $P(1.5)$ & $D_{C}$ & $P(0.25)$ & $P(1.5)$ \\
\hline${ }_{\Lambda}^{3} \mathrm{H}$ & - & - & - & 9.2 & 0.4 & 1.3 & 8.5 & 0.5 & 1.1 \\
\hline${ }_{\Lambda}^{4} \mathrm{H}$ & 55. & 11. & 23. & 48. & 9.3 & 18. & 22. & 4.1 & 8.3 \\
\hline${ }_{\Lambda}^{6} \mathrm{H}$ & - & - & - & 1.3 & 0.2 & 0.1 & 0.3 & $<0.1$ & $<0.1$ \\
\hline${ }_{\Lambda}^{6} \mathrm{He}$ & 36. & 4.8 & 5.6 & 6.9 & 0.9 & 1.1 & 1.1 & 0.1 & 0.2 \\
\hline
\end{tabular}

Table 2: Statistical model predictions for the probabilities $D_{C}\left({ }_{\Lambda}^{A} Z, E_{\text {exc }}\right)$ of forming a hyperfragment ${ }_{\Lambda}^{A} Z$ from the compound hypernucleus $C$ with an excitation energy $E_{\text {exc }}$ and for the product $P(x)=D_{C} \times S(x) \times B r$ of $D_{C}$ with the stopping probability $S(x)$ for Li target foils of thickness $x$ and the two-body $\pi^{-}$decay branching ratio $\operatorname{Br}\left({ }_{\Lambda}^{A} Z\right)=\Gamma\left({ }^{A}(Z+1)+\pi^{-}\right) / \Gamma$ of the hyperfragment. Values are given for three different $E_{\text {exc }}$. The maximum $P$ value for each process is emphasized in bold.

For the fragmentation from Be targets, three possible scenarios were considered: A compound hypernucleus, a compound hypernucleus after proton knockout, and a compound hypernucleus after neutron knockout. For the three scenarios different distributions possible hyperfragments were obtained, with several fragmentation channels already open at an excitation energy of $E_{\mathrm{exc}}=20 \mathrm{MeV}$, while others need $30 \mathrm{MeV}$ or more. From the distributions it is seen that above $E_{\text {exc }} \sim 30 \mathrm{MeV}$ a rich spectrum of hyperfragments is possible. When stopping and two-body pionic decay probabilities were taken into account ${ }_{\Lambda}^{4} \mathrm{H}$ becomes the dominant isotope in all three scenarios because of its exceptionally large two-body branching ratio. 

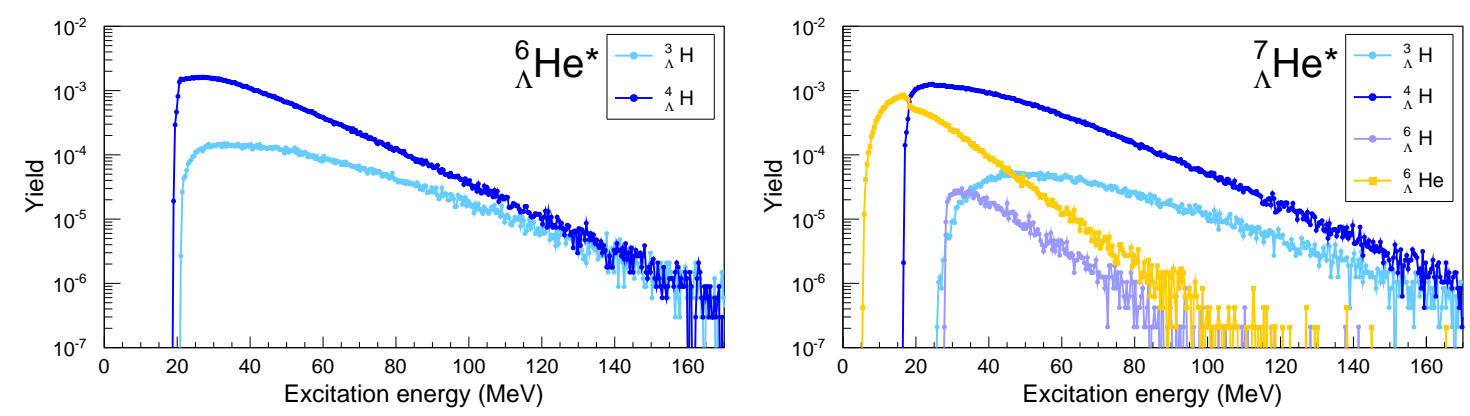

Figure 2: Statistical model predictions for the relative hyperfragmentation yields with subsequent two-body $\pi^{-}$decay from rest in $250 \mu \mathrm{m}$ thick ${ }^{6} \mathrm{Li}$ and ${ }^{7} \mathrm{Li}$ targets as a function of the excitation energy of the compound hypernucleus. The $\Lambda$ binding energies $B_{\Lambda}\left({ }_{\Lambda}^{6} \mathrm{He}\right)=4.18 \mathrm{MeV}$ and $B_{\Lambda}\left({ }_{\Lambda}^{7} \mathrm{He}\right)=5.36 \mathrm{MeV}$ were considered as the minimum available energy for the break-up process.

\begin{tabular}{l|c|ccc|c|c}
\hline \hline & & \multicolumn{3}{|c|}{${ }^{9} \mathrm{Be}$ target } & ${ }^{7}$ Li target & ${ }^{6}$ Li target \\
\hline${ }_{\Lambda}^{A} \mathrm{Z}$ & $p_{\pi}(\mathrm{MeV} / c)$ & ${ }_{\Lambda}^{9} \mathrm{Li}^{*}$ & ${ }_{\Lambda}^{8} \mathrm{Li}^{*}$ & ${ }_{\Lambda}^{8} \mathrm{He}^{*}$ & ${ }_{\Lambda}^{7} \mathrm{He}^{*}$ & ${ }_{\Lambda}^{6} \mathrm{He}^{*}$ \\
\hline${ }_{\Lambda}^{3} \mathrm{H}$ & $114.37 \pm 0.08$ & 0.56 & 1.18 & 0.67 & 1.49 & 2.48 \\
${ }_{\Lambda}^{4} \mathrm{H}$ & $132.87 \pm 0.06$ & 3.56 & 3.74 & 7.51 & 12.61 & 8.81 \\
${ }_{\Lambda}^{6} \mathrm{H}$ & $135.13 \pm 1.52$ & 0.03 & $<0.01$ & 0.23 & 0.10 & - \\
${ }_{\Lambda}^{6} \mathrm{He}$ & $108.47 \pm 0.18$ & 2.44 & 1.25 & 1.47 & 3.53 & - \\
${ }_{\Lambda}^{7} \mathrm{He}$ & $114.97 \pm 0.15 \pm 0.17$ & 2.12 & 0.44 & 1.35 & - & - \\
${ }_{\Lambda}^{8} \mathrm{He}$ & $116.50 \pm 1.08$ & 0.04 & - & - & - & - \\
${ }_{\Lambda}^{7} \mathrm{Li}$ & $108.11 \pm 0.05$ & 1.54 & 1.68 & - & - & - \\
${ }_{\Lambda}^{8} \mathrm{Li}$ & $124.20 \pm 0.05$ & 0.85 & - & - & - & - \\
\hline \hline
\end{tabular}

Table 3: Statistical model predictions for the excitation energy integrated probabilities in $\%$ of forming a hyperfragment ${ }_{\Lambda}^{A} Z$ with subsequent two-body $\pi^{-}$decay at rest in the fragmentation of an excited hypersystem. The decay momenta $p_{\pi}$ for possible hyperfragments are given with their known uncertainties. For the fragmentation of beryllium target nuclei three possible scenarios were modeled: an excited ${ }_{\Lambda}^{9} \mathrm{Li}^{*}$ compound hypernucleus, an initial ${ }_{\Lambda}^{8} \mathrm{Li}^{*}$ compound system after neutron knockout, and an initial ${ }_{\Lambda}^{8} \mathrm{He}^{*}$ system after proton knockout. For the fragmentation of lithium target nuclei the predictions for direct $\Lambda$ captures in ${ }^{7} \mathrm{Li}$ or ${ }^{6} \mathrm{Li}$ nuclei are given. Note, that the predictions for ${ }^{6-8}{ }_{\Lambda} \mathrm{He}$ have large uncertainties due to the unknown two-body $\pi^{-}$decay branching ratios.

Table 3 lists the excitation energy integrated production probability per compound nucleus in percent. Because of the exceptionally large two-body branching ratio, ${ }_{\Lambda}^{4} \mathrm{H}$ becomes the isotope with maximum probabilities in all three scenarios. All other hypernuclei decays are produced with at least a factor of two lower rates and, therefore, could not be detected with sufficient statistics in the first experiments. According to these calculations, the production probability of hypertriton is a factor of 3-11 lower compared to ${ }_{\Lambda}^{4} \mathrm{H}$. This suggests, that simply increasing the running time for the measurement will not be sufficient to observe hypertriton decays.

Note, that in addition to these hypernuclei from the fragmentation of excited compound hy- 
pernuclei, also a direct ground states production is possible. However, the process can not be calculated within this approach. In any case, the non-observation of ${ }_{\Lambda}^{9} \mathrm{Li}$ in the experiments of 2012 and 2014 indicates that the direct process may be less likely.

\section{Lithium Target for Hypertriton Production}

In the 2012 and 2014 experiments the MAMI beam with an energy of $1.5 \mathrm{GeV}$ and an current of $20-50 \mu \mathrm{A}$ was incident on a beryllium foil of either $125 \mu \mathrm{m}$ or $250 \mu \mathrm{m}$ thickness. The ${ }^{9} \mathrm{Be}$ target was tilted by $54^{\circ}$ with respect to the beam direction to minimize the energy straggling of negative pions leaving the target in direction of the spectrometers. A significantly thicker target with the same arrangement would have caused a too large spread of the pion momenta. Also, an increase of the luminosity could not be achieved by an increase of the beam current, because neutrons originating from the beam dump are the dominant source for random triggers. Even a factor of two increase of the beam intensity would have raised the count rate caused by background coincidences beyond an acceptable level.
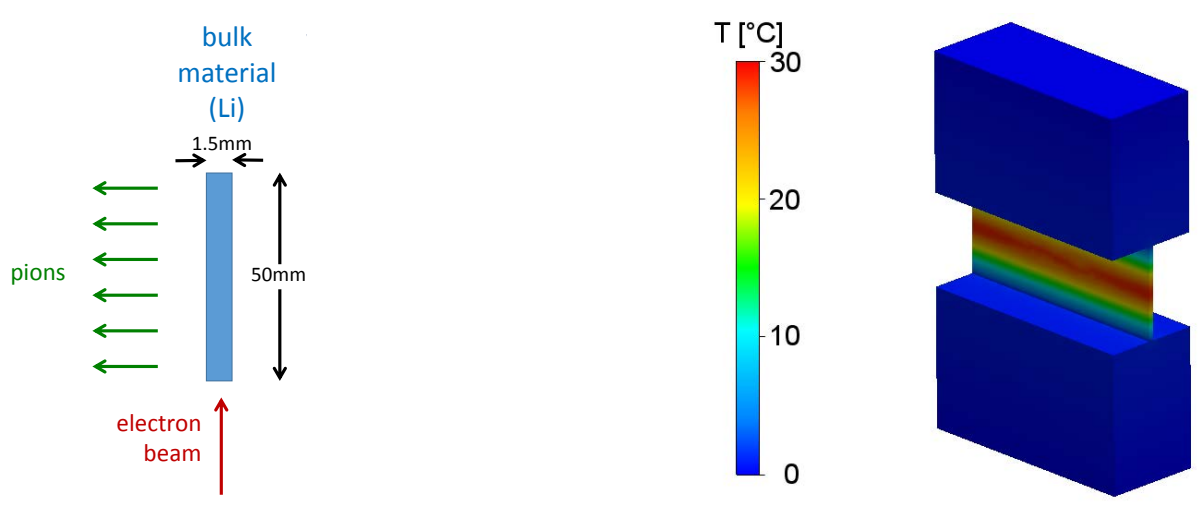

Figure 3: Left: Schematic view of a possible Li target and detection geometry to be employed in the hypertriton measurement (view from top; not to scale). Right: Expected temperature distribution in a $1 \mathrm{~mm}$ thick block of $\mathrm{Li}$ inside a cooling frame, while an electron beam of $5 \mu \mathrm{A}$ current, vertically wobbled by $\Delta y= \pm 2 \mathrm{~mm}$, is entering the target.

Fortunately, the excellent quality and stability of the MAMI beam, combined with a low density target material enables a novel target geometry: Instead of a tilted target, the beam could be incident at a $1 \mathrm{~mm}$ wide surface of a $50 \mathrm{~mm}$ long target block, see Fig. 3 (left) for a schematic view of the target and detection geometry. Pions that will be emitted at $\pm 90^{\circ}$ with respect to the beam direction toward the spectrometers, would traverse only a thin target layer, keeping their momentum spread acceptable low. Such a target geometry allows a gain in luminosity by about one order of magnitude at a significantly lower beam current. The reduced beam intensity will also diminish the background count rate caused by neutrons and will furthermore guarantee more stable running conditions at MAMI.

Lithium with its low density of $\rho=0.534 \mathrm{~g} / \mathrm{cm}^{3}$ for natural $\mathrm{Li}$ and $\rho=0.460 \mathrm{~g} / \mathrm{cm}^{3}$ for ${ }^{6} \mathrm{Li}$ as compared to $\rho=1.848 \mathrm{~g} / \mathrm{cm}^{3}$ for Be is a unique candidate for the novel target geometry mentioned above. A solid-state Li target was tested for use in experiments requiring high beam intensities. For this purpose, Li material was embedded in a water-cooled copper frame and sensors were attached 

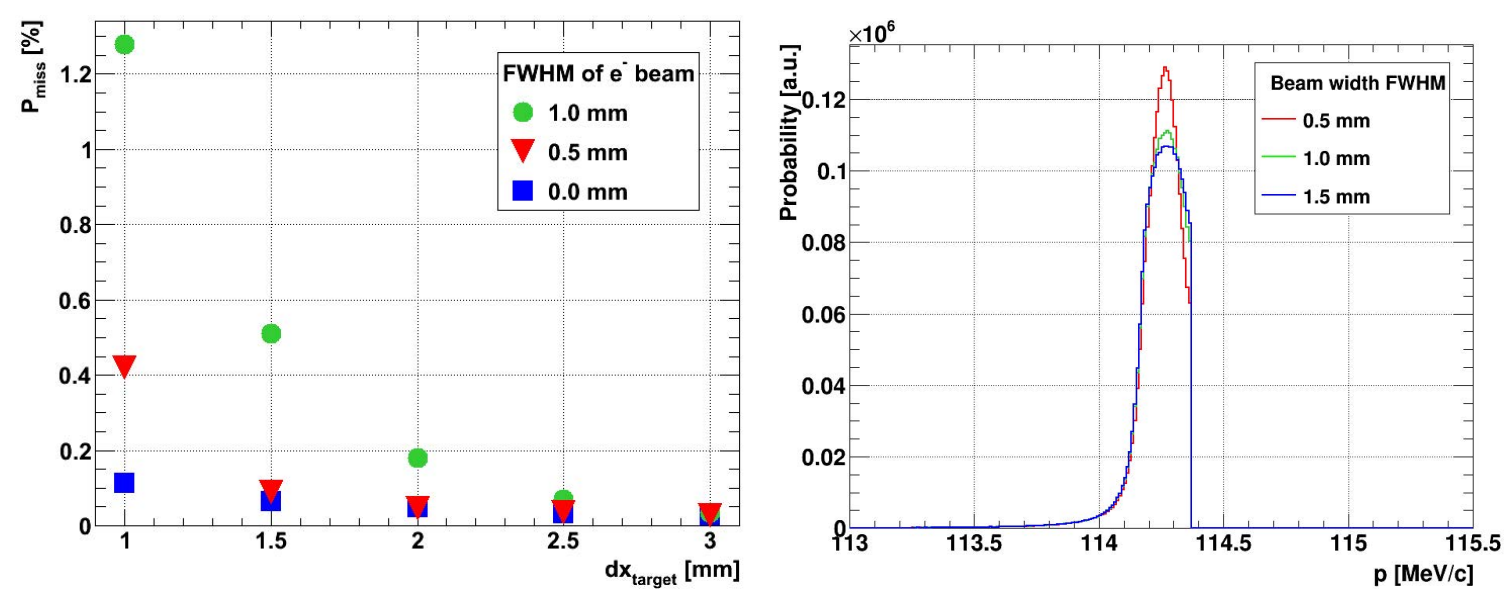

Figure 4: Left: GEANT simulations for the percentage $P_{\text {miss }}$ of beam electrons entering a $50 \mathrm{~mm}$ long Li block and scattered transversely out of the target as a function of the target width $d x_{\text {target }}$ and for different Gaussian profile beam widths. Right: Expected momentum distribution of pions from hypertriton weak decays in a $1 \mathrm{~mm}$ thick Li target for different Gaussian profile beam widths.

to monitor temperatures. With this target setup beam currents of more than $20 \mu \mathrm{A}$ were possible without reaching critical temperatures near the melting point of $\mathrm{Li}$ at about $180^{\circ} \mathrm{C}$. Fig. 3 (right) shows the preliminary design of a $\mathrm{Li}$ target for the hypertriton measurement and the simulated temperature profile across the Li block. For this simulation, a beam current of $5 \mu \mathrm{A}$ entering the target and a temperature of the cooling frame of $0{ }^{\circ} \mathrm{C}$ was adopted. The electron beam was wobbled by $\Delta y= \pm 2 \mathrm{~mm}$ in vertical direction. The maximum temperature of about $30^{\circ} \mathrm{C}$ in the target center was found to be far below the melting point.

The number of electrons scattered transversely out of the target is very small. Even in case of a $1 \mathrm{~mm}$ wide target and a FWHM beam width of $1 \mathrm{~mm}$, nearly $99 \%$ of the electrons entering the target will traverse the complete $50 \mathrm{~mm}$ target thickness, see Fig. 4 (left). The stability of the luminosity will be monitored by the single rates in the spectrometers, and a large transverse beam size helps to avoid luminosity fluctuations in case of small beam movements. In case of such a $1 \mathrm{~mm}$ wide Li target, the momentum line of the pions from hypertriton decays will be broadened to $\delta p_{\pi} \approx 200 \mathrm{keV} / c$ (FWHM) as shown by the GEANT simulations in Fig. 4 (right).

\section{Hypertriton Experiment at MAMI}

In order to reduce model dependencies, rate and background estimates for a hypertriton experiment at MAMI can be made relative to the successful observation of ${ }_{\Lambda}^{4} \mathrm{H}$ in 2012 and 2014. Since the $\Lambda$ production cross section scales with $Z^{0.7}$ [30], a factor of 0.82 has to be employed for a $\mathrm{Li}$ target as compared to the Be target. The lower pion momentum of $114 \mathrm{MeV} / c$ compared to $133 \mathrm{MeV} / c$ from ${ }_{\Lambda}^{4} \mathrm{H}$ reduces the survival probability for pions in the spectrometers by a factor 0.9. The statistical model calculations indicate that the hypertriton fragmentation and subsequent weak pionic two-body decay at rest is of the order of $0.70-0.33$ with respect to the ${ }_{\Lambda}^{4} \mathrm{H}$ yield from the Be target, depending on the assumed formation process. This will allow to determine the peak position with a statistical uncertainty below $20 \mathrm{keV} / c$. 


\section{Conclusions}

The study of the binding energy of the $\Lambda$ in the lightest hypernucleus, the hypertriton, can reveal important details of the strong nucleon-hyperon interaction. At the Mainz Microtron MAMI the high-resolution spectroscopy of decay-pions in strangeness electro-production can be used to extract the binding energy of hypertriton. Statistical decay calculations suggest that lithium is the optimal target material to observe hypertriton decays under relative clean conditions with only few other light hyperfragments being produced.

\section{Acknowledgments}

This work was supported in part by the Federal State of Rhineland-Palatinate and by the Deutsche Forschungsgemeinschaft (DFG) with the Collaborative Research Center SFB 1044.

\section{References}

[1] D. H. Davis, Hypernuclei - The early days, Nucl. Phys. A 547 (1992) 369-378.

[2] D. H. Davis, 50 years of hypernuclear physics - I. The early experiments, Nucl. Phys. A 754 (2005) $3 c-13 c$.

[3] A1 Collaboration, A. Esser et al., Observation of ${ }_{\Lambda}^{4} \mathrm{H}$ hyperhydrogen by decay-pion spectroscopy in electron scattering, Phys. Rev. Lett. 114 (2015) 232501.

[4] M. Jurić et al., A new determination of the binding-energy values of the light hypernuclei $(A \leq 15)$, Nucl. Phys. B 52 (1973) 1-30.

[5] G. Bohm et al., A determination of the binding-energy values of light hypernuclei, Nucl. Phys. B 4 (1968) 511-526.

[6] W. Gajewski et al., A compilation of binding energy values of light hypernuclei, Nucl. Phys. B 1 (1967) 105-113.

[7] HKS Collaboration, T. Gogami et al., High resolution spectroscopic study of ${ }_{\Lambda}^{10} \mathrm{Be}$, Phys. Rev. $\mathbf{C} 93$ (2016) 034314.

[8] O. Hashimoto and H. Tamura, Spectroscopy of $\Lambda$ hypernuclei, Prog. Part. Nucl. Phys. 57 (2006) 564-653.

[9] E. Botta, T. Bressani and A. Feliciello, On the binding energy and the charge symmetry breaking in A $\leq 16$ \-hypernuclei, Nucl. Phys. A 960 (2017) 165-179.

[10] A1 Collaboration, F. Schulz et al., Ground-state binding energy of ${ }_{\Lambda}^{4} \mathrm{H}$ from high-resolution decay-pion spectroscopy, Nucl. Phys. A 954 (2016) 149-160.

[11] R. H. Dalitz and G. Rajasekharan, The spins and lifetimes of the light hypernuclei, Phys. Lett. 1 (1962) 58-60.

[12] M. Rayet and R. H. Dalitz, The lifetime of ${ }^{3} H_{\Lambda}$, Nuovo Cim. A 46 (1966) 786-794.

[13] B. W. Downs and R. H. Dalitz, Analysis of the $\Lambda$-hypernuclear three-body systems, Phys. Rev. 114 (1959) 593-602.

[14] STAR Collaboration, B. I. Abelev et al., Observation of an antimatter hypernucleus, Science 328 (2010) 58-62. 
[15] HYPHI Collaboration, C. Rappold et al., Hypernuclear spectroscopy of products from ${ }^{6} \mathrm{Li}$ projectiles on a carbon target at 2 AGeV, Nucl. Phys. A 931 (2013) 170-184.

[16] ALICE Collaboration, J. Adam et al., ${ }_{\Lambda}^{3} \mathrm{H}$ and $\frac{3}{\Lambda} \overline{\mathrm{H}}$ production in $\mathrm{Pb}-\mathrm{Pb}$ collisions at $\sqrt{s_{\mathrm{NN}}}=2.76$ TeV, Phys. Lett. B 754 (2016) 360-372.

[17] Proceedings of the 12th International Conference on Hypernuclear and Strange Particle Physics (HYP2015), Sendai, Japan, 7-12 Sept. 2015, JPS Conf. Proc. 17, 2017.

[18] E864 Collaboration, T. A. Armstrong et al., Production of ${ }_{\Lambda}^{3} \mathrm{H}$ and ${ }_{\Lambda}^{4} \mathrm{H}$ in central $11.5 \mathrm{GeV} / \mathrm{c} \mathrm{Au}+\mathrm{Pt}$ heavy ion collisions, Phys. Rev. C 70 (2017) 024902.

[19] STAR Collaboration, Y. Zhu et al., Beam energy scan on hypertriton production and lifetime measurement at RHIC STAR, Nucl. Phys. 904-905 (2013) 551c-554c.

[20] J. Steinheimer, K. Gudima, A. Botvina, I. Mishustin, M. Bleicher and H. Stöcker, Hypernuclei, dibaryon and antinuclei production in high energy heavy ion collisions: Thermal production vs. coalescence, Phys. Lett. B 714 (2012) 85-91.

[21] A. Esser, Zerfallspionenspektroskopie leichter Hyperkerne in der Elektroproduktion, doctoral thesis, Johannes Gutenberg-Universität Mainz, Germany, 2014.

[22] F. Schulz, Präzisionsmessung der $\Lambda$-Separationsenergie von ${ }_{\Lambda}^{4} \mathrm{H}$ am Mainzer Mikrotron, doctoral thesis, Johannes Gutenberg-Universität Mainz, Germany, 2016.

[23] CLAS Collaboration, P. Ambrozewicz et al., Separated structure functions for the exclusive electroproduction of $K^{+} \Lambda$ and $K^{+} \Sigma^{0}$ final states, Phys. Rev. $\mathbf{C} 75$ (2007) 045203.

[24] E91-016 Collaboration, F. Dohrmann et al., Quasifree $\Lambda$, $\Sigma^{0}$, and $\Sigma^{-}$electroproduction from ${ }^{1,2} \mathrm{H}$, ${ }^{3,4} \mathrm{He}$, and carbon, Phys. Rev. C 76 (2007) 054004.

[25] H. Tamura et al., Compound-hypernucleus interpretation on ${ }_{\Lambda}^{4} \mathrm{H}$ formation probabilities in stopped-K $K^{-}$absorption, Phys. Rev. C 40 (1989) 483-486.

[26] H. Bandō, T. Motoba and J. Žofka, Production, structure and decay of hypernuclei, Int. J. Mod. Phys. A 5 (1990) 4021-4198.

[27] A. Sanchez Lorente, A. S. Botvina and J. Pochodzalla, Production of excited double hypernuclei via Fermi breakup of excited strange systems, Phys. Lett. B 697 (2011) 222-228.

[28] E. Fermi, High-energy nuclear events, Prog. Theor. Phys. 5 (1950) 570-583.

[29] J. P. Bondorf, A. S. Botvina, A. S. Ilinov, I. N. Mishustin and K. Sneppen, Statistical multifragmentation of nuclei, Phys. Rept. 257 (1995) 133-221.

[30] H. Yamazaki et al., The ${ }^{12} \mathrm{C}\left(\gamma, K^{+}\right)$reaction in the threshold region, Phys. Rev. C 52 (1995) R1157-R1160. 